\title{
Perfil Teórico das Publicações sobre Orçamento em Periódicos Nacionais e Internacionais (2003 a 2013)
}

\begin{abstract}
Zandra Cristina Lima Silva Queiroz
Mestrado em Ciências Contábeis pela Universidade Federal de Uberlâdia - UFU Professora na Pontifícia Universidade Católica de Minas Gerais - PUC Minas Av. Dom José Gaspar, 500. Coração Eucarístico. Belo Horizonte/MG, CEP: 30535-901

E-mail: zandracristina@zipmail.com.br

Rodrigo Fernandes Malaquias Doutorado em Administração de Empresas pela Fundação Getúlio Vargas - FGV/SP Professor na Universidade Federal de Uberlândia - UFU Av. João Naves de Ávila, 2121. Santa Mônica. Uberlândia/MG. CEP: 38408-100 E-mail: rodrigofmalaquias@yahoo.com.br
\end{abstract}

\section{RESUMO}

O objetivo principal deste estudo foi analisar o conteúdo teórico dos artigos acadêmicos publicados sobre o tema orçamento nos principais periódicos nacionais e internacionais no período de 2003 a 2013. Para tanto, realizou-se um levantamento bibliográfico com abordagem quantitativa, que contou com uma amostra final de 45 artigos. Na etapa quantitativa, foi aplicada a estatística descritiva, a análise de regressão multivariada e a análise de regressão logística. Os principais resultados evidenciaram que o número de referências dos estudos apresentou relação positiva e significativa com o conteúdo teórico presente na introdução e no referencial teórico. Além disso, observou-se que não houve relação significativa entre as características dos artigos e a contribuição teórica (dummy) presente nas considerações finais dos trabalhos. Uma vez que a abordagem utilizada neste artigo não foi encontrada em outro estudo já publicado, entende-se que uma nova discussão pode ser iniciada, podendo também auxiliar pesquisadores na seleção de artigos para fundamentar pesquisas futuras a serem desenvolvidas nesta área.

Palavras-chave: Orçamento. Planejamento. Contribuição Teórica.

\section{Theoretical Profile of Publications on Budget in Brazilian and International} Journals (2003-2013)

\section{ABSTRACT}

The aim of this study was to analyze the theoretical content of academic articles published on the topic budget in major national and international journals in the period 2003-2013. It was performed a bibliographic study with quantitative approach, which included a final sample of 45 articles. In the quantitative phase, descriptive statistics, multivariate regression analysis and logistic regression analysis were applied. The main 
Perfil Teórico das Publicações sobre Orçamento em Periódicos Nacionais e Internacionais (2003 a

results indicated that the number of references of the studies showed a positive and significant relationship with the academic content that is present in the introduction and theoretical framework. Moreover, it was observed that there was no significant relationship between the characteristics of the articles and theoretical contribution (dummy variable) in the final considerations of the papers. Since the approach used in this article was not found in a previously published study, it is understood that a new thread can be started and may also assist researchers in the selection of articles to support future research to be undertaken in this area.

Key-words: Budget. Planning. Theoretical Contribution.

\section{INTRODUÇÃO}

O esforço de unir os conceitos contábeis como ferramentas importantes para a tomada de decisões constitui-se em um dos pilares da contabilidade gerencial atualmente (LEITE et al., 2008). Para Horngren, Sundem e Stratton (2004, p. 8), "os gestores beneficiam-se quando a contabilidade fornece informações que os ajudam a planejar e a controlar as operações da organização" e que a contabilidade gerencial "compreende uma série de atividades em um ciclo de planejamento e controle". $\mathrm{Na}$ literatura da contabilidade gerencial, conceituações sobre o orçamento e a sua utilização encontram-se inseridas dentro de compreensões de uma ferramenta que apóia os gestores em suas funções de planejamento e controle (ANTHONY; GOVINDARAJAN, 2002; HORNGREN; SUDEN; STRATTON 2004; ATKINSON et al., 2000).

Para Covaleski et al. (2006), o orçamento é usado para muitos propósitos, incluindo o planejamento e a coordenação de atividades de uma organização, alocando recursos, motivando os funcionários e expressando conformidade com as normas sociais. Simas, Costa e Moritz (2008) afirmam que além de ser um instrumento de planejamento e controle, os orçamentos desempenham papel importante para coordenação das atividades dos gestores junto às demais áreas, pois por meio de orçamentos os gestores podem acompanhar os líderes de cada área, controlar o seu 
Perfil Teórico das Publicações sobre Orçamento em Periódicos Nacionais e Internacionais (2003 a

Zandra Cristina Lima Silva Queiroz, Rodrigo Fernandes Malaquias

desempenho junto às metas estabelecidas e verificar se estão alinhados com os objetivos organizacionais.

Existem autores que enfatizam que o orçamento surge como uma relevante ferramenta de controle gerencial, a qual explora a competência estratégica de contadores e gestores (FAURÉ; ROULEAU, 2011) e também inferem que dentro do contexto atual, em que as empresas estão inseridas em grandes mudanças, os sistemas de gestão devem possuir ferramentas que as auxiliem a alcançar os objetivos organizacionais, fornecendo informações sobre desempenho e resultados (BARBOSA FILHO; PARISI, 2006). Em contrapartida, outros autores argumentam que, apesar de historicamente desempenhar um papel central nos sistemas de controle de gestão, o orçamento tem sido objeto de críticas, que enfatizam a necessidade de evolução com a adição de novas estratégias que ajudem as empresas a competirem eficazmente e de forma acirrada em um ambiente que trabalha com informações rápidas (LIBBY; LINDSAY, 2010).

Neste sentido, consideram-se relevantes os estudos que produzam conhecimentos, por meio dos quais a comunidade científica possa inserir, complementar e contestar as contribuições anteriormente dadas, enriquecendo os estudos de um determinado tema, conforme sustentam Moura, Dallabona e Lavarda (2012). Segundo os referidos autores, percebe-se que grande parte dos estudos realizados sobre o tema em questão tende a evidenciar os assuntos atuais e recentes desenvolvidos por pesquisadores das diversas áreas, e com a proliferação de pesquisas a comunidade acadêmica se enriquece, principalmente com estudos bibliométricos que buscam identificar os aspectos relevantes.

Neste contexto, Lunkes, Feliu e Rosa (2011) observaram que a área de orçamento necessita de trabalhos que avaliem a produção científica relacionada ao tema. Isto inclui estudos sobre os tópicos investigados e as metodologias utilizadas, além das características das revistas, artigos, autores e citações. Portanto, estudar como essas características são descritas na literatura, principalmente em revistas, é importante para o conhecimento do nível de desenvolvimento e inovação. Assim, é 
Perfil Teórico das Publicações sobre Orçamento em Periódicos Nacionais e Internacionais (2003 a

Zandra Cristina Lima Silva Queiroz, Rodrigo Fernandes Malaquias

possível proporcionar informações importantes para os estudiosos do tema e a aplicação prática desses nas organizações.

Teoria, de acordo com Whetten (2003), deve possuir quatro elementos essenciais, sendo eles: "o que", "como", "por que" e "quem, onde e quando". Segundo Moura, Dallabona e Lavarda (2012) são poucos os estudos que contribuem para o aprimoramento teórico acerca da temática relacionada com orçamento. Diante dessa escassez, os referidos autores desenvolveram pesquisa sobre o perfil bibliométrico dos artigos publicados sobre o tema Orçamento nos Congressos USP de Controladoria e Contabilidade, EnANPAD, Congresso ANPCONT e Congresso Brasileiro de Custos, no período de 2005 a 2009, apontando possíveis lacunas na literatura nacional sobre a temática orçamento.

Sobre a relevância do tema, pode-se destacar que com a implementação de orçamentos, o processo de tomada de decisão poderá considerar aspectos relevantes das atividades, alinhá-las às estratégias da empresa e permitir antecipar possíveis resultados operacionais, garantindo precisão e controle dos processos organizacionais (SIMAS; COSTA; MORITZ, 2008).

Neste contexto, este trabalho apresentou como objetivo analisar o conteúdo teórico dos artigos acadêmicos publicados sobre o tema orçamento nos principais periódicos nacionais e internacionais no período de 2003 a 2013. As etapas desenvolvidas para atingir o objetivo geral foram: i) selecionar os periódicos para análise dos artigos; ii) desenvolver um instrumento de coleta de dados; iii) analisar a contribuição teórica dos trabalhos, bem como analisar as características relacionadas com esses estudos; iv) verificar potenciais relações entre as características dos trabalhos e as contribuições teóricas apresentadas por eles.

Simas, Costa e Moritz (2008) afirmam que a relevância dos estudos acerca do tema em periódicos nacionais e internacionais nos últimos dez anos, justifica-se pelo fato de que o sistema orçamentário é uma condição essencial para o sucesso empresarial, pois permite por meio de previsão, identificar o caminho que a empresa irá percorrer através da visualização antecipada das atividades que serão desempenhadas 
Perfil Teórico das Publicações sobre Orçamento em Periódicos Nacionais e Internacionais (2003 a

Zandra Cristina Lima Silva Queiroz, Rodrigo Fernandes Malaquias

no período projetado. Além disso, a discussão apresentada no decorrer do trabalho pode auxiliar pesquisas futuras na formalização e/ou identificação da contribuição apresentada pelos trabalhos a serem analisados e também para uso referencial dos principais autores revelados nesse estudo (FARIA et al., 2011).

\section{REFERENCIAL TEÓRICO}

No decorrer dos estudos avaliados para a realização desta pesquisa, vários conceitos de orçamento foram elencados. Destacam-se os descritos no Quadro 1. Observou-se consenso entre os autores quanto aos conceitos e a sua importância, os quais ressaltam a necessidade de adaptação à realidade de mercado e sua evolução. 
Zandra Cristina Lima Silva Queiroz, Rodrigo Fernandes Malaquias

\begin{tabular}{|c|c|}
\hline Autor & Conceito de Orçamento \\
\hline $\begin{array}{l}\text { Hansen, Otley e Van Der Stede (2003) } \\
\text { Frezatti (2004), } \\
\text { Simas, Costa e Moritz (2008), } \\
\text { Bornia e Lunkes (2009), } \\
\text { Etemadi et al. (2009), } \\
\text { Heinzmann e Lavarda (2012), } \\
\text { Lima Filho, Bruni e Sampaio (2012). }\end{array}$ & $\begin{array}{l}\text { O orçamento é um instrumento que auxilia o planejamento e o } \\
\text { controle de uma organização e define as ações específicas e } \\
\text { identificadas de cada responsável pela execução das atividades nele } \\
\text { discriminadas. }\end{array}$ \\
\hline Chenhall (2003). & $\begin{array}{l}\text { O orçamento é definido como um conjunto de práticas da } \\
\text { contabilidade gerencial. }\end{array}$ \\
\hline Vilas Boas e Jones (2005). & $\begin{array}{l}\text { O orçamento está inserido no conceito de planejamento e } \\
\text { antecipação dos resultados esperados no futuro de um determinado } \\
\text { período. }\end{array}$ \\
\hline Covaleski et al. (2006). & $\begin{array}{l}\text { O orçamento está relacionado a praticamente todos os aspectos da } \\
\text { contabilidade gerencial, tais como: a análise do custo contábil, da } \\
\text { responsabilidade, do desempenho e da medição, compensação, } \\
\text { alocação de recursos, planejamento e coordenação nas atividades de } \\
\text { uma organização. Menciona também relacionamento com motivação } \\
\text { de funcionários. }\end{array}$ \\
\hline Merchant (2007). & $\begin{array}{l}\text { O orçamento está inserido no planejamento e controle de curto } \\
\text { prazo e é a união do fluxo de informação, processos e } \\
\text { procedimentos administrativos. }\end{array}$ \\
\hline Bin e Castor (2007). & $\begin{array}{l}\text { O orçamento está inserido no conceito de planejamento e controle } \\
\text { racional. É a forma de consubstanciar as decisões de planejamento e } \\
\text { estratégias organizacionais. }\end{array}$ \\
\hline Leite et al. (2008). & $\begin{array}{l}\text { O orçamento visa o atendimento da missão organizacional e } \\
\text { estratégias da empresa, permite foco nas operações e finanças, } \\
\text { antecipa problemas, sinaliza metas e objetivos e contribui para a } \\
\text { tomada de decisão. }\end{array}$ \\
\hline Almeida et al . (2009). & $\begin{array}{l}\text { O orçamento é parte do processo de gestão que traduz em valores } \\
\text { monetários o que foi planejado pelas unidades operacionais e } \\
\text { administrativas da empresa. }\end{array}$ \\
\hline Vanzella e Lunkes (2009). & $\begin{array}{l}\text { O orçamento funciona como medida de vendas, rentabilidade, custos } \\
\text { e despesas departamentais. Também é utilizado para atingir metas } \\
\text { financeiras pré-definidas. }\end{array}$ \\
\hline Libby e Lindsay (2010). & $\begin{array}{l}\text { Orçamentos são ferramentas de controle, no entanto, recentemente } \\
\text { tornaram-se alvo de críticas e debate considerável. }\end{array}$ \\
\hline Souza e Lavarda (2011). & $\begin{array}{l}\text { O orçamento é um artefato de planejamento e controle utilizado para } \\
\text { proporcionar segurança, acompanhar as metas estabelecidas e } \\
\text { avaliar o desempenho dos gestores. }\end{array}$ \\
\hline
\end{tabular}


Zandra Cristina Lima Silva Queiroz, Rodrigo Fernandes Malaquias

\begin{tabular}{|l|l|}
\hline Autor & Conceito de Orçamento \\
\hline Moura, Dallabona e Lavarda (2012). & $\begin{array}{l}\text { O orçamento é utilizado para controlar o uso de recursos } \\
\text { disponíveis, avaliar o desempenho de suas atividades, auxiliar no } \\
\text { processo decisório e na implementação de planos estratégicos. }\end{array}$ \\
\hline Lunkes, Feliu e Rosa (2011). & $\begin{array}{l}\text { O orçamento é um plano de metas e recursos que direciona a } \\
\text { execução, através da comparação do previsto com o realizado, } \\
\text { permitindo por meio da analise das diferenças implementar as } \\
\text { medidas de ajustes. }\end{array}$ \\
\hline Church, Hannan e Kuang (2012). & $\begin{array}{l}\text { O orçamento está vinculado ao planejamento e controle de } \\
\text { atividades e alocação de recursos. }\end{array}$ \\
\hline Souza e Lunkes (2013). & $\begin{array}{l}\text { O orçamento representa um instrumento nas fases de planejamento e } \\
\text { controle que orienta a organização quanto à avaliação do } \\
\text { desempenho e motivação pessoal. }\end{array}$ \\
\hline
\end{tabular}

Quadro 1 - Conceitos de orçamento

Fonte: Autores mencionados no quadro.

Diante dos conceitos apresentados pelos autores citados, observa-se um consenso quanto à associação do orçamento como um artefato ou instrumento utilizado nas etapas de planejamento e controle de atividades e que também é utilizado como guia na execução das tarefas previamente estabelecidas.

No que se refere à importância, os autores esboçam que o orçamento ao compor as práticas da contabilidade gerencial, se destaca como um referencial para os gestores no processo de tomada de decisão. Apesar de possuir críticas quanto à necessidade de orçar ou não, o orçamento ainda é um modelo amplamente estudado e utilizado.

Nos primórdios do orçamento, o mesmo era utilizado como ferramenta de previsão de recursos, com controle por meio de dados contábeis. A partir de 1919 emergiu a necessidade do acréscimo de novas variáveis que permitissem uma maior assertividade quanto à previsão, maior flexibilidade, direcionamento e por fim, no ano 2000, uma projeção flexível e descentralizada (BORNIA; LUNKES, 2007). Segundo Bornia e Lunkes (2009, p.39), 
Perfil Teórico das Publicações sobre Orçamento em Periódicos Nacionais e Internacionais (2003 a

Zandra Cristina Lima Silva Queiroz, Rodrigo Fernandes Malaquias

a necessidade de orçar é tão antiga quanto a humanidade. Os homens das cavernas precisavam prever a necessidade de comida para os longos invernos. Assim, desenvolveram práticas antigas de orçamento. Há vestígios de práticas orçamentárias formais até mais antigas que a origem do dinheiro. O orçamento sempre fez parte dos sistemas de administração pública das principais civilizações ocidentais.

No contexto atual, os estudos sobre a contabilidade gerencial, em especial o orçamento, podem apresentar diferentes perspectivas como ferramenta de planejamento e controle, relação com teorias sociais como: teoria contingencial (CHENHALL, 2003) e teoria organizacional (LIMA FILHO; BRUNI; SAMPAIO 2012), além de estudos de caso que visam constatar a sua utilização, bem como suas variações (CARMONA; NYVER, 2011; BORNIA; LUNKES, 2007; FREZATTI, 2005; ALMEIDA et al., 2009; SOUZA; LAVARDA, 2012; SIMAS; COSTA; MORITZ, 2008; FAURÉ ROULEAU, 2011; VANZELLA; LUNKES, 2006; BARBOSA FILHO; PARISI, 2006; BIN; CASTOR, 2007). Nesse estudo o foco está centrado na produção científica, em especial periódicos nacionais e internacionais. O Quadro 2, a seguir, destaca alguns dos estudos já realizados de 2003 a 2013. 
Zandra Cristina Lima Silva Queiroz, Rodrigo Fernandes Malaquias

\begin{tabular}{|c|c|}
\hline Autores & Breve descrição do estudo \\
\hline $\begin{array}{l}\text { Hansen, Otley e Van Der } \\
\text { Stede (2003) }\end{array}$ & $\begin{array}{l}\text { Por meio da observação das práticas gerenciais, estudaram a utilização do balanced } \\
\text { scorecard como forma de controle baseado em dados contábeis e não financeiros. }\end{array}$ \\
\hline Frezatti (2005) & $\begin{array}{l}\text { Estudou a abordagem Beyond Budgeting, e verificou que a ausência do orçamento pode } \\
\text { acarretar problemas além de evidenciar outros que não existiriam sem esta nova } \\
\text { aborgadem. }\end{array}$ \\
\hline Merchant (2007) & $\begin{array}{l}\text { Evidenciou que há utilização maior do orçamento em empresas maiores e que, em todas } \\
\text { as empresas estudadas, a formalização dos processos de orçamento é bem recebida pelos } \\
\text { gerentes. }\end{array}$ \\
\hline $\begin{array}{l}\text { Simas, Costa e Moritz } \\
\text { (2008) }\end{array}$ & $\begin{array}{l}\text { Na amostra estudada, observaram que as empresas não possuem estrutura de sistema de } \\
\text { orçamento. Sua elaboração é feita pela alta administração, principalmente por planilhas } \\
\text { eletrônicas. }\end{array}$ \\
\hline Bornia e Lunkes (2009) & $\begin{array}{l}\text { Demonstraram resultados positivos quanto a utilização do balanced scorecard e sua } \\
\text { associação ao orçamento. Constataram que a integração de ambos melhora a gestão e } \\
\text { controle através de indicadores de desempenho. }\end{array}$ \\
\hline Etemadi et al. (2009) & $\begin{array}{l}\text { Indica que valores culturais devem ser considerados na aplicação de vários sistemas e } \\
\text { ferramentas de gestão de uma empresa. }\end{array}$ \\
\hline Libby e Lindsay (2010) & A maioria das empresas utiliza os orçamentos para fins de controle. \\
\hline $\begin{array}{l}\text { Lima Filho, Bruni e } \\
\text { Sampaio (2012) }\end{array}$ & $\begin{array}{l}\text { Este estudo aponta que as relações existentes entre as variáveis idade, gênero e formação } \\
\text { no processo de controle orçamentário e que somente a variável gênero houve uma } \\
\text { relação significativa. }\end{array}$ \\
\hline
\end{tabular}

Quadro 2 - Exemplos de estudos já realizados sobre orçamento (2003 a 2013) Fonte: Autores mencionados no quadro.

O exposto no Quadro 2 coopera com a identificação de fatores que influenciam a prática orçamentária, os quais analisam técnicas como o Balanced Scorecard e Beyond Budget e também o relacionamento das técnicas com características comportamentais e estruturais das empresas e seus reflexos na utilização do orçamento.

\section{ASPECTOS METODOLÓGICOS}

Esta pesquisa caracteriza-se como descritiva, pois o objetivo é descrever as características de uma determinada população ou um fenômeno e estabelecer relação entre elas, principalmente por meio de técnicas específicas de coleta de dados (GIL, 1999). Quanto à abordagem do problema a pesquisa é quantitativa. Richardson (1999) 
Perfil Teórico das Publicações sobre Orçamento em Periódicos Nacionais e Internacionais (2003 a

afirma que a abordagem quantitativa caracteriza-se pelo emprego da quantificação tanto nas modalidades de coleta das informações, quanto no tratamento delas por meio de técnicas estatísticas. O autor destaca sua importância ao ter a intenção de garantir a precisão dos resultados, evitar distorções de análise e interpretação, possibilitando uma margem de segurança quanto às inferências feitas.

Quanto ao procedimento é do tipo levantamento bibliográfico, que tem o objetivo de explicar, conhecer e analisar, por meio de referências em documentos publicados, as contribuições teóricas e científicas de um referido tema (CERVO; BERVIAN, 1983).

A coleta de dados foi realizada por meio de uma seleção de artigos que apresentaram o termo orçamento (budget) no título, no resumo ou nas palavras-chave, publicados em periódicos nacionais e internacionais no período de 2003 a 2013, resultando em 45 artigos para análise.

No processo de seleção dos periódicos foram escolhidos, inicialmente, dois grupos: um grupo com periódicos nacionais e outro com periódicos internacionais. Para análise dos periódicos nacionais foi considerada a lista de revistas disponíveis no sistema QUALIS/CAPES da área de Administração, Ciências Contábeis e Turismo com qualificação mínima de B2. Dentre os periódicos internacionais foram selecionados aqueles que além de constarem na citada lista do QUALIS/CAPES, com referida qualificação mínima, também apresentaram fator de impacto mínimo. Os periódicos que tiveram artigos selecionados para o estudo estão elencados a seguir:

I. periódicos nacionais: BBR; Contabilidade Vista \& Revista; Contabilidade, Gestão e Governança; Contextus (Fortaleza); RAC; RAE; Revista Contabilidade \& Finanças; Revista de Ciências da Administração (CAD/UFSC); Revista de Contabilidade e Organizações; Revista Universo Contábil.

II. periódicos internacionais: Accounting, Organizations and Society; Advances in Accounting; Critical Perspectives on Accounting; International Journal of Accounting Information Systems; Journal of Accounting Education; Management Accounting Research; The British Accounting Review. 
Perfil Teórico das Publicações sobre Orçamento em Periódicos Nacionais e Internacionais (2003 a

Para a análise dos artigos foi elaborada uma planilha com os seguintes campos: autor, periódico, ano, número de páginas, quantidade de páginas na introdução, referencial teórico, metodologia e resultados, número de referências e número de referências em periódicos. Estes itens já foram utilizados em estudos bibliométricos anteriores como o de Moura, Dallabona e Lavarda (2012). Além disso, nesta planilha, quatro campos armazenaram o que se refere à contribuição teórica dos trabalhos (Quadro 3) com padrões de resposta previamente definidos.

\begin{tabular}{|c|c|}
\hline Descrição Campo & Padrão de Respostas \\
\hline $\begin{array}{l}\text { Apresentou contribuição teórica nas } \\
\text { considerações finais? }\end{array}$ & $\begin{array}{l}\text { Variável dummy. Foi atribuído o valor } 0 \text { caso o trabalho não } \\
\text { apresentasse a contribuição teórica e o valor } 1 \text { para aqueles } \\
\text { com contribuição apresentada no trabalho. }\end{array}$ \\
\hline Nota para a introdução do artigo & $\begin{array}{l}\text { Foram atribuídas notas, na escala de } 0 \text { a } 2 \text {, referentes à } \\
\text { introdução dos artigos (clareza, concatenação de idéias, } \\
\text { apresentação da relevância do tema e justificativas). }\end{array}$ \\
\hline Checklist: & Padrão de resposta, para cada um dos itens do Checklist: \\
\hline O autor evidenciou: "o que"? & 0: não evidenciou \\
\hline O autor evidenciou: "como"? & 1: evidenciou parcialmente \\
\hline O autor evidenciou: "por que"? & 2: evidenciou \\
\hline O autor evidenciou: "quem, onde e quando"? & \\
\hline
\end{tabular}

Quadro 3 - Componentes do instrumento de coleta de dados para avaliar a contribuição teórica Fonte: Baseado em Moura, Dallabona e Lavarda (2012).

O score obtido com os quatro itens do checklist serviu de base para a construção de um índice, cujo valor foi dividido pela nota máxima possível: 8. Esta variável passou a ser chamada de "Teoria", e seu valor varia entre 0 e 1 . Para esta variável, notas próximas de zero indicam que na introdução ou no referencial teórico houve pouca ou nenhuma ocorrência de discussão teórica nos termos que podem ser observados no trabalho de Whetten (2003). Notas próximas de 1 indicam estudos em que a teoria foi identificada conforme as recomendações do referido autor. 
Quanto à análise quantitativa, primeiramente utilizaram-se recursos da estatística descritiva. Para mensurar uma potencial relação entre as características dos artigos e o índice da variável "Teoria" (Checklist), foi utilizada a análise de regressão múltipla (HAIR et. al., 2005). Já para a variável que se refere à contribuição teórica (que é representada por uma dummy), foi aplicada a análise de regressão logística para avaliar a relação entre as variáveis do estudo e a contribuição teórica presente nas considerações finais. De acordo com Fávero et al. (2009, p. 441), a análise de regressão logística permite "investigar a relação entre variáveis explicativas, métricas e não métricas, e uma variável dependente categórica binária".

\section{RESULTADOS}

Os Gráficos 1 e 2, a seguir, apresentam, respectivamente, a quantidade de artigos, por ano, selecionados para o estudo e a frequência relativa (em percentual) dos trabalhos segregados por tipo de abordagem: qualitativa, quantitativa ou ambas.

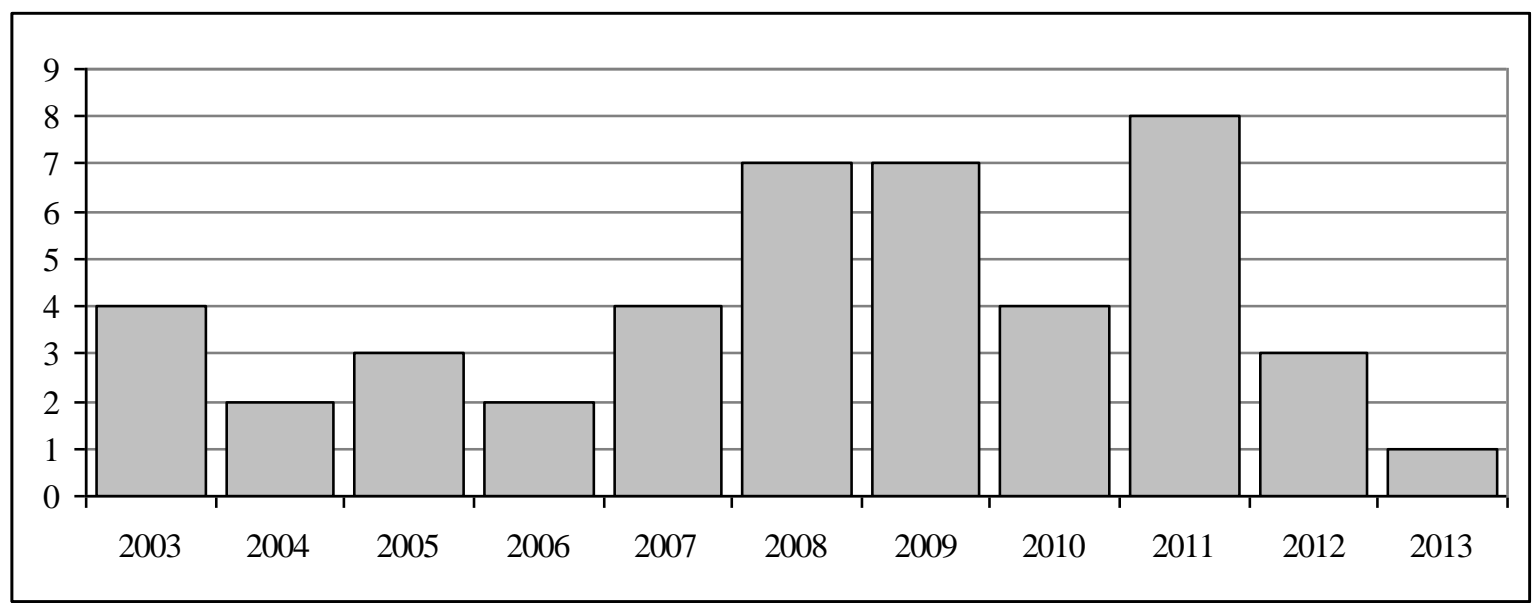

Gráfico 1 - Quantidade de artigos selecionados, por ano Fonte: Dados da pesquisa.

A maior concentração de artigos se deu no ano de 2011, sendo que o período de 2008 e 2009 também contou com considerável volume de artigos publicados sobre 
Perfil Teórico das Publicações sobre Orçamento em Periódicos Nacionais e Internacionais (2003 a

orçamento nos periódicos avaliados. Foram coletados 22 artigos provenientes de periódicos nacionais e 23 internacionais, representando uma amostra de 45 artigos acadêmicos.

A abordagem utilizada nos artigos pode ser visualizada no Gráfico 2.
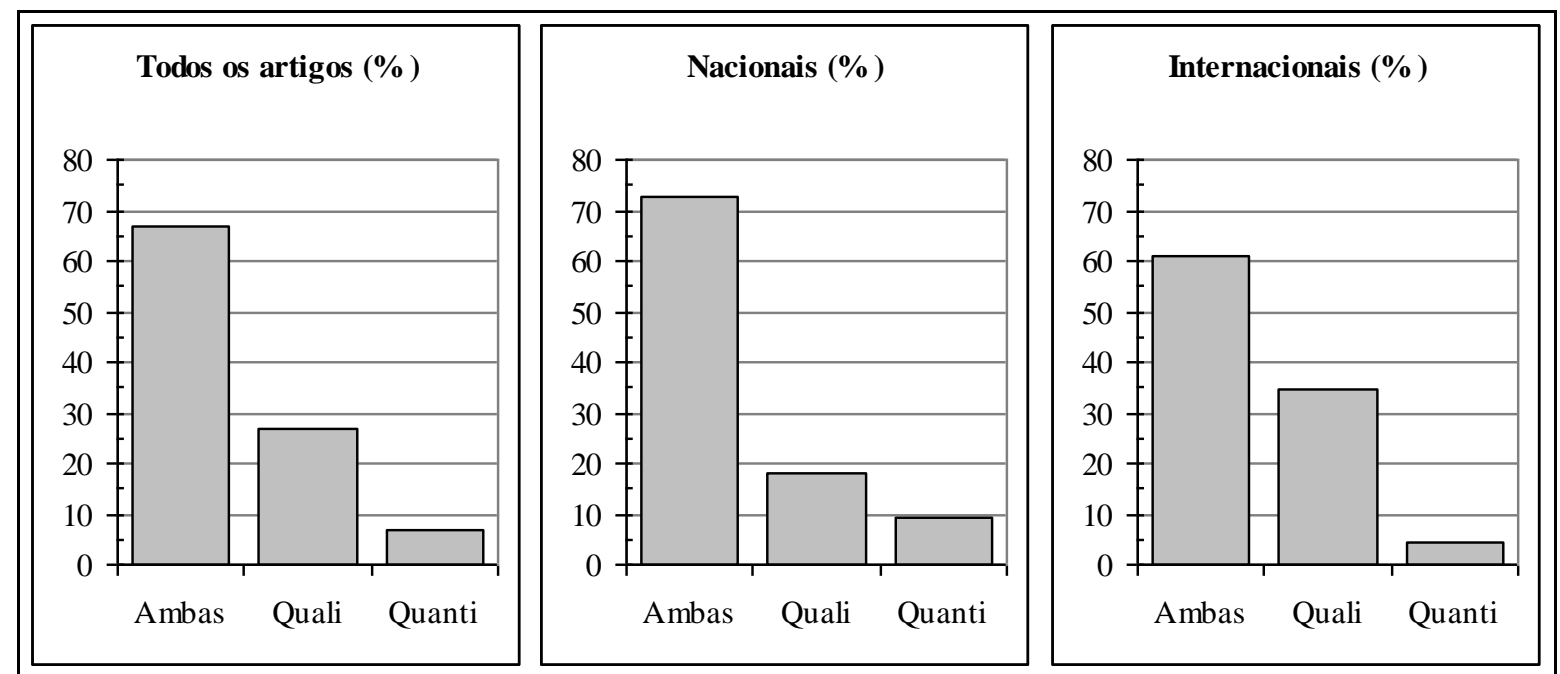

Gráfico 2 - Percentual de trabalhos, por tipo de abordagem Fonte: Dados da pesquisa.

O tipo de abordagem utilizado pelos autores com maior frequência é a combinação entre pesquisa qualitativa e quantitativa, sejam em estudos nacionais ou internacionais, conforme sustenta o Gráfico 2.

O passo seguinte consistiu na elaboração da estatística descritiva pra as variáveis quantitativas do estudo, conforme apresentado na Tabela 1. A última coluna da referida tabela indica o nível de significância para um teste t entre as médias das variáveis para periódicos nacionais e internacionais. 
Perfil Teórico das Publicações sobre Orçamento em Periódicos Nacionais e Internacionais (2003 a

Zandra Cristina Lima Silva Queiroz, Rodrigo Fernandes Malaquias

Tabela 1 - Estatística descritiva das variáveis do estudo

\begin{tabular}{|c|c|c|c|c|c|c|c|}
\hline Variáveis & Nac./Intern. & $\mathbf{n}$ & Média & Desv. Pad. & Mínimo & Máximo & Signif. \\
\hline \multirow{2}{*}{$\mathrm{n}^{\circ}$ páginas total } & Nacional & 22 & 19,409 & 7,607 & 9,000 & 43,000 & \multirow{2}{*}{0,751} \\
\hline & Internacional & 23 & 18,652 & 8,233 & 7,000 & 41,000 & \\
\hline \multirow{2}{*}{$\mathrm{n}^{\circ}$ páginas introdução } & Nacional & 22 & 1,727 & 0,922 & 0,500 & 4,000 & \multirow{2}{*}{0,123} \\
\hline & Internacional & 23 & 1,348 & 0,681 & 0,500 & 3,500 & \\
\hline \multirow{2}{*}{$\mathrm{n}^{\circ}$ páginas ref. teórico } & Nacional & 22 & 5,523 & 3,072 & 0,000 & 14,000 & \multirow{2}{*}{0,283} \\
\hline & Internacional & 23 & 7,696 & 8,885 & 0,000 & 34,000 & \\
\hline \multirow{2}{*}{$\mathrm{n}^{\mathrm{o}}$ páginas metodologia } & Nacional & 22 & 1,977 & 1,658 & 0,000 & 5,000 & \multirow{2}{*}{0,975} \\
\hline & Internacional & 23 & 2,000 & 2,892 & 0,000 & 11,000 & \\
\hline \multirow{2}{*}{$\mathrm{n}^{\circ}$ páginas resultados } & Nacional & 22 & 5,773 & 3,261 & 0,000 & 15,000 & \multirow{2}{*}{0,142} \\
\hline & Internacional & 23 & 4,196 & 3,771 & 0,000 & 13,000 & \\
\hline \multirow{2}{*}{$\mathrm{n}^{\circ}$ páginas consid. finais } & Nacional & 22 & 1,250 & 0,551 & 0,500 & 2,500 & \multirow{2}{*}{0,308} \\
\hline & Internacional & 23 & 1,522 & 1,113 & 0,500 & 6,000 & \\
\hline \multirow{2}{*}{$\mathrm{n}^{\circ}$ referências - geral } & Nacional & 22 & 32,455 & 19,997 & 7,000 & 87,000 & \multirow{2}{*}{0,004} \\
\hline & Internacional & 23 & 67,565 & 51,254 & 6,000 & 237,000 & \\
\hline \multirow{2}{*}{$\mathrm{n}^{\circ}$ referências - periódicos } & Nacional & 22 & 15,136 & 18,093 & 0,000 & 67,000 & \multirow{2}{*}{0,000} \\
\hline & Internacional & 23 & 64,739 & 51,675 & 20,000 & 237,000 & \\
\hline \multirow{2}{*}{ Nota Introdução } & Nacional & 22 & 1,864 & 0,351 & 1,000 & 2,000 & \multirow{2}{*}{0,103} \\
\hline & Internacional & 23 & 1,652 & 0,487 & 1,000 & 2,000 & \\
\hline \multirow{2}{*}{ Contrib. Teórica (dummy) } & Nacional & 22 & 0,909 & 0,294 & 0,000 & 1,000 & \multirow{2}{*}{0,682} \\
\hline & Internacional & 23 & 0,870 & 0,344 & 0,000 & 1,000 & \\
\hline \multirow{2}{*}{ Teoria (Checklist) } & Nacional & 22 & 0,898 & 0,217 & 0,250 & 1,000 & \multirow{2}{*}{0,351} \\
\hline & Internacional & 23 & 0,826 & 0,286 & 0,000 & 1,000 & \\
\hline
\end{tabular}

Fonte: Dados da pesquisa.

Conforme indicam os dados da Tabela 1, as notas atribuídas às variáveis: Introdução dos artigos; Contribuição Teórica nas considerações finais e Teoria (medida pelo checklist) foram equivalentes para periódicos nacionais e internacionais a um nível de significância de 5\%. A introdução destes estudos apresentou nota média acima de 1,5 , estando próxima de 2 , indicando que os trabalhos avaliados apresentam (em média) de maneira adequada uma contextualização sobre o assunto pesquisado, suas justificativas e relevância do estudo, além de conterem parágrafos concatenados. 
Para as variáveis relativas ao número de páginas, o tamanho dedicado ao texto dos trabalhos analisados não difere significativamente entre artigos nacionais $\mathrm{e}$ internacionais, pois o teste t não foi significativo para estas variáveis ao nível de $5 \%$. As diferenças significativas estiveram presentes no número de referências utilizadas, uma vez que os artigos publicados em periódicos internacionais utilizam mais que o dobro de referências dos artigos nacionais. Além disso, o nível de utilização de referências provenientes de outros periódicos é mais intenso nos artigos internacionais, que registraram, em média, mais que o quádruplo de referências provenientes de artigos de periódicos.

Para verificar se esta diferença apresenta alguma relação com o nível de teoria presente nos artigos e também com a contribuição teórica proporcionada por eles, foram realizadas as análises de regressão. A Tabela 2 resume os resultados da análise de regressão múltipla, na qual a variável dependente foi a nota para a Teoria presente no artigo (Checklist). Com exceção da variável "Contribuição Teórica (Dummy)", todas as variáveis disponíveis na Tabela 1 foram incluídas no modelo, utilizando-se o método stepwise para escolha do melhor grupo de variáveis explicativas.

Tabela 2 - Análise de regressão entre as variáveis do estudo e a Teoria presente no artigo (Checklist)

\begin{tabular}{lcccc}
\hline Variáveis & beta & Std. Error & Estat. t & Signif. \\
\hline Nota Introdução & 0,341 & 0,071 & 4,841 & 0,000 \\
$\mathrm{n}^{\text {o referências - geral }}$ & 0,002 & 0,001 & 2,387 & 0,022 \\
\hline r-quadrado ajustado: & & 0,366 & & \\
signific. Estat. F & 0,000 & & \\
Resíduos Padroniz. Normais? & $\operatorname{sim}$ & & \\
\hline
\end{tabular}

Notas: método aplicado na análise de regressão multivariada: stepwise, para seleção do melhor modelo com base nas variáveis independentes relacionadas na Tabela 1. Normalidade dos resíduos padronizados avaliada com base no teste de Kolmogorov-Smirnov, a um nível de 5\% de significância.

Número de observações: 45.

Fonte: Dados da pesquisa.

Os resultados obtidos com a análise de regressão indicaram que as variáveis que apresentaram relação significativa com a teoria contida nos artigos (introdução e 
referencial teórico) foram a nota dada à introdução do estudo e também o número de referências utilizadas no estudo, independentemente do tipo destas referências. Ou seja, estes resultados indicam que dos 45 artigos analisados, aqueles que possuem introdução elaborada com maior cuidado e que, ao mesmo tempo, os autores consultaram um número maior de referências para a fundamentação do texto, apresentam maior probabilidade de conter aspectos teóricos (WHETTEN, 2003) em seu conteúdo.

Conter teoria no corpo da introdução ou do referencial teórico não seria mais do que o necessário para trabalhos acadêmicos já publicados (embora nem todos os artigos cumpram plenamente com os requisitos exigidos para se ter teoria nos trabalhos). Desta forma, a análise seguinte consistiu na identificação de fatores relacionados com a contribuição teórica presente nas considerações finais dos artigos. Em outras palavras, na análise seguinte, pretendeu-se verificar características de trabalhos acadêmicos que estão significativamente relacionadas com a contribuição dos artigos para a discussão teórica na área de orçamento.

Os testes foram formalizados com base na análise de regressão logística, contudo os modelos testados não apresentaram betas significativos. Uma das justificativas está relacionada com o reduzido número de artigos que não apresentou contribuição teórica nas considerações finais (apenas 5). Desta forma, com base nos testes realizados e, principalmente, considerando-se a base de dados que foi construída, a inferência que se pode fazer é a seguinte: artigos provenientes de periódicos com QUALIS/CAPES mínimo de B2 ou que, mesmo não estando na lista do QUALIS/CAPES, apresentem considerável fator de impacto, apresentaram contribuição teórica para a área de orçamento. Consequentemente, estes estudos representam fontes adequadas de consultas para estudos futuros a serem realizados neste campo, o que está em linha com a classificação destes periódicos. 
Perfil Teórico das Publicações sobre Orçamento em Periódicos Nacionais e Internacionais (2003 a

Zandra Cristina Lima Silva Queiroz, Rodrigo Fernandes Malaquias

\section{CONSIDERAÇÕES FINAIS}

O presente estudo teve por objetivo analisar o conteúdo teórico dos artigos acadêmicos publicados sobre o tema orçamento nos principais periódicos nacionais e internacionais, no período de 2003 a 2013. Para a realização de testes quantitativos e potenciais relações de dependência entre as variáveis, foram analisadas: a quantidade de referências dos trabalhos, a quantidade de páginas na introdução, no referencial teórico, na metodologia e nos resultados, o número de referências e o número de referências em periódicos. O estudo foi conduzido por meio de um levantamento bibliográfico, com a realização de análise quantitativa multivariada de dados.

Foram selecionados 45 artigos publicados sobre o tema, tendo sido verificado que os estudos apresentaram significativo volume de contribuição teórica sobre o assunto (apenas 5 artigos não apresentaram contribuição teórica nas considerações finais). A distribuição da contribuição teórica entre artigos nacionais e internacionais foi equivalente, conforme evidenciado na Tabela 1, em que ambos os tipos de artigos apresentaram frequência próxima de $90 \%$, pois as contribuição teórica nas considerações finais foi medida por meio de uma variável dummy.

Com base nos resultados da análise de regressão multivariada, observou-se uma associação positiva e estatisticamente significativa entre o número de referências (variável independente), o conteúdo da introdução dos estudos (variável independente) e o conteúdo teórico presente no trabalho (variável dependente), nos termos considerados por Whetten (2003) a respeito do assunto. Estes resultados indicam que artigos com maior quantidade de referências e com introdução elaborada com cuidado tendem a apresentar adequadamente aspectos de teoria no corpo do texto.

Por outro lado, não foram identificadas variáveis significativamente correlacionadas com a contribuição teórica presente nas considerações finais dos artigos, fato que pode ser explicado pela base de dados ter sido formada por estudos que já contam com elevado nível de qualidade em sua elaboração (por autores) e avaliação (por editores e revisores dos periódicos). 
Perfil Teórico das Publicações sobre Orçamento em Periódicos Nacionais e Internacionais (2003 a

Como a abordagem utilizada neste estudo não foi identificada em outra pesquisa já publicada, entende-se que ela permite iniciar a discussão sobre variáveis que afetam a contribuição de estudos acadêmicos, permitindo também estender o estudo de Whetten (2003) no que se refere a contribuição teórica. Para pesquisas futuras sugerese a aplicação de outros parâmetros bibliométricos, a identificação dos autores das referências mais utilizadas, bem como a ampliação da amostra para um número maior de revistas nacionais e internacionais. Talvez seja oportuno incluir na base de dados dissertações e teses defendidas no âmbito da Contabilidade Gerencial.

\section{REFERÊNCIAS}

ALMEIDA, L. B., MACHADO, E. A., RAIFUR, L., \& NOGUEIRA, D. R. A (2009). Utilização do orçamento como ferramenta de apoio à formulação de estratégia, de controle e de interatividade: um estudo exploratório nas cooperativas agropecuárias da região sul do Brasil. Contabilidade Vista \& Revista, v. 20, n. 3, p. 65-99.

ANTHONY, R.N.; GOVINDARAJAN, V. (2002). Sistemas de controle gerencial. McGraw Hill Brasil.

ATKINSON, A. A; BANKER, R. B; K. R.S; YOUNG, S. M. (2000). Contabilidade Gerencial. São Paulo: Atlas.

BARBOSA FILHO, F; PARISI, C. (2006). Análise da aderência ao modelo Beyond Budgeting Round Table: O CASO SADIA SA. Revista Universo Contábil, v. 2, n. 1, p. 26-42.

BIN, D; CASTOR, B.V.J. (2007). Racionalidade e política no processo decisório: estudo sobre orçamento em uma organização estatal. Revista de Administração Contemporânea, v. 11, n. 3, p. 35-56.

BORNIA, A. C.; LUNKES, R.J. (2009). Uma contribuição à melhoria do processo orçamentário. Contabilidade Vista \& Revista, v. 18, n. 4, p. 37-59.

CALLEGARI-JACQUES, S. (2003). Bioestatística: princípios e aplicações. Porto Alegre: Artemed.

CARMONA, S; IYER, G; RECKERS, P. (2011). The impact of strategy communications, incentives and national culture on balanced scorecard implementation. Advances in Accounting, v. 27, n. 1, p. 62-74. 
Perfil Teórico das Publicações sobre Orçamento em Periódicos Nacionais e Internacionais (2003 a

Zandra Cristina Lima Silva Queiroz, Rodrigo Fernandes Malaquias

CERVO, A. L; BERVIAN, P. A. (1983). Metodologia Científica. (3 ed.). São Paulo: McGraw-Hill do Brasil.

CHAPMAN, C.S.; KIHN, L. (2009). Information system integration, enabling control and performance. Accounting, organizations and society, v. 34, n. 2, p. 151-169.

CHENHALL, R. H. (2003). Management control systems design within its organizational context: findings from contingency-based research and directions for the future. Accounting, organizations and society, v. 28, n. 2, p. 127-168.

CHURCH, B.K.; HANNAN, R. L.; KUANG, X. J. (2012). Shared interest and honesty in budget reporting. Accounting, Organizations and Society, v. 37, n. 3, p. 155-167.

COVALESKI, M. et al. (2006). Budgeting research: three theoretical perspectives and criteria for selective integration. Handbooks of Management Accounting Research, v. 2, p. 587-624.

ETEMADI, $H$. et al. (2009). Culture, management accounting and managerial performance: focus Iran. Advances in Accounting, v. 25, n. 2, p. 216-225.

FARIA, J. A. et al. (2011). A assimetria da informação na elaboração do orçamento: uma análise da produção científica nos periódicos internacionais entre 2005 e 2009. Contabilidade Vista \& Revista, v. 22, n. 2, p. 43-65.

FÁVERO, Luiz Paulo et al. (2009). Análise de Dados: modelagem multivariada para tomada de decisões. Rio de Janeiro: Elsevier.

FREZATTI, F. (2004). Além do orçamento: existiria alguma chance de melhoria do gerenciamento? Brazilian Business Review, v. 1, n. 2, p. 122-140.

FREZATTI, F. (2005). Beyond Budgeting: inovação ou resgate de antigos conceitos do orçamento empresarial. RAE - Revista de Administração de Empresas, n. 2, p. 23-33.

FAURÉ, B.; ROULEAU, L. (2011). The strategic competence of accountants and middle managers in budget making. Accounting, Organizations and Society, v. 36, n. 3, p. 167182.

GIL, A. C. (1999). Metodologia e técnicas de pesquisa social. Antonio Carlos Gil/Fabiane Clemente. São Paulo: Atlas. 
Perfil Teórico das Publicações sobre Orçamento em Periódicos Nacionais e Internacionais (2003 a

Zandra Cristina Lima Silva Queiroz, Rodrigo Fernandes Malaquias

HANSEN, S. C.; OTLEY, D. T.; VAN DER STEDE, W. (2003). Practice developments in budgeting: an overview and research perspective. Journal of Management Accounting Research, Forthcoming.

HAIR, J. F.; ANDERSON, R. E.; TATHAM, R. L.; BLACK, W. C. (2005). Análise Multivariada de Dados. Tradução de Adonai Schulup Sant'Anna e Anselmo Chaves Neto. (5 ed.). Porto Alegre: Bookman.

HEINZMANN, L. M; LAVARDA, C. E. F. (2012). Cultura organizacional e o processo de Planejamento e Controle Orçamentário. Revista de Contabilidade e Organizações, v. 5, n. 13 , p. 4-19.

HORNGREN, C. T.; SUDEM, G. L.; STRATTON, W. O. (2004). Contabilidade gerencial. 12. ed. São Paulo: Prentice Hall.

LEITE, R.M. et al. (2008). Orçamento empresarial: levantamento da produção científica no período de 1995 a 2006. Revista de Contabilidade e Finanças. São Paulo, v. 19, n. 47 , p. $56-72$.

LIBBY, T.; LINDSAY, R. M. (2010). Beyond budgeting or budgeting reconsidered? A survey of North-American budgeting practice. Management Accounting Research, v. 21, n. 1 , p. 56-75.

LIMA FILHO, R.; BRUNI, A.; SAMPAIO, M. (2012). A Influência do Gênero, Idade e Formação na Presença de Heurísticas em Decisões de Orçamento: Um Estudo Quase experimental. Revista Universo Contábil, v. 8, n. 2, p. 103-117.

LUNKES, R. J; FELIU,V. R. F; ROSA, F. S. (2011). Pesquisa sobre o orçamento na Espanha: um estudo bibliométrico das publicações em contabilidade. Revista Universo Contábil. v.7, n.3, p. 112-132, Jul./Set.

MERCHANT, K. A. (2007). O modelo do sistema de orçamento corporativo: influências no comportamento e no desempenho gerencial. Revista de Contabilidade $e$ Organizações, v. 1, n. 1, p. 104-121.

MOURA, G. D.; DALLABONA, L. F.; LAVARDA, C. E. F. (2012). Perfil dos estudos sobre o tema orçamento publicado em congressos brasileiros de 2005 a 2009. Contabilidade Vista \& Revista, v. 23, n. 1, p. 97-125.

RICHARDSON, Roberto Jarry. (1999). Pesquisa Social: métodos e técnicas. São Paulo: Atlas. 
SILVA, A. J.; TOLEDO FILHO, J. R.; PINTO, J. (2009). Análise bibliométrica dos artigos sobre controladoria publicados em periódicos dos programas de pós-graduação em Ciências Contábeis recomendados pela Capes. Revista da Associação Brasileira de Custos. Rio Grande do Sul, v. 4, n. 1.

SIMAS, F. K.; COSTA, A.M.; MORITZ, G. de O. (2008). Um estudo sobre a organização e o desenvolvimento do processo orçamentário em empresas de tecnologia de Florianópolis. Revista de Ciências da Administração, v. 10, n. 21, p. 197-219.

SOUZA, R. P. S; LAVARDA, C. E. F. (2012). Avaliação do Beyond Budgeting como ferramenta de planejamento e controle na visão dos gestores de IES. Contextus Revista Contemporânea de Economia e Gestão, v. 9, n. 2, p. 69-78.

SOUZA, P.; LUNKES, R. J. (2013). Práticas de Orçamento de Capital: Um estudo em empresas hoteleiras de Florianópolis (SC). Revista Universo Contábil, v. 9, n. 1, p. 2845.

VANZELLA, C.; LUNKES, R. J. (2009). Orçamento Baseado em Atividades: um estudo de caso em empresa distribuidora de energia elétrica. Contabilidade Vista \& Revista, v. 17 , n. 1, p. 113-132.

VILAS BOAS, A. A.; JONES, G.D.C. (2005). Planejamento Financeiro e Controle Orçamentário: um estudo de caso em uma empresa industrial. Contextus - Revista Contemporânea de Economia e Gestão, v. 3, n. 1, p. 25-34.

WHETTEN, D. A. (2003). O que constitui uma contribuição teórica? RAE, v. 43, n. 3, p. 69-73. Jul/Set.

Data de Submissão: 20/12/2013

Data de Aceite: 23/03/2014 